\title{
Confidence Intervals for the Population Correlation Coefficient $\rho$
}

\author{
Shipra Banik ${ }^{1, *}$ and B.M. Golam Kibria ${ }^{2}$
}

${ }^{1}$ Department of Physical Sciences, Independent University, Bangladesh, Dhaka 1229, Bangladesh

${ }^{2}$ Department of Mathematics and Statistics, Florida International University, Miami, FL 33199, USA

\begin{abstract}
Computing a confidence interval for a population correlation coefficient is very important for researchers as it gives an estimated range of values which is likely to include an unknown population correlation coefficient. This paper studied some confidence intervals for estimating the population correlation coefficient $\rho$ by means of a Monte Carlo simulation study. Data are randomly generated from several bivariate distributions with a various values of sample sizes. Assessment measures such as coverage probability, mean width and standard deviation of the width are selected for performances evaluation. Two real life data are analyzed to demonstrate the application of the proposed confidence intervals. Based on our findings, some good confidence intervals for a population correlation coefficient are suggested for practitioners and applied researchers.
\end{abstract}

Keywords: Bivariate distribution, Bootstrapping, Correlation coefficient, Confidence interval, Simulation study.

\section{INTRODUCTION}

One of the most often used statistical measures is the correlation coefficient which determines the degree of linear relationship between two quantitative variables. To understand the concept of correlation, let us consider a real life situation. Suppose a health care specialist is interested to find the familial aggregation of blood pressure. It is well known that children whose parents have high blood pressure be likely to have higher blood pressure than their peers. A first step for a health care specialist should be to assess whether there is a positive relationship exists between children and parents blood pressure. One way of expressing this relationship is to calculate a correlation coefficient between blood pressure of parents and children over a group of families. There are two kinds of estimators (point and confidence interval) are available in literature to estimate the population correlation coefficient $(\rho)$. In statistics, the Pearson product-moment correlation coefficient, referred as Pearson's $r$ (proposed by Fisher (1921) [1] is commonly used to quantity the linear relationship between two quantitative variables (say) $X$ and $\mathrm{Y}$. In our example, $r$ is the sample correlation coefficient between the blood pressure of parents and children of some randomly selected families. It gives a value between -1 and +1 inclusive, where +1 represents a perfect positive correlation, 0 is no correlation and -1 represents a perfect negative correlation. The correlation coefficient has been widely used in science as a measure of the degree of linear dependence between two variables

*Address correspondence to this author at the Department of Physical Sciences, Independent University, Bangladesh, Dhaka 1229, Bangladesh; Tel: 8402056-76; Fax: 8401991; E-mail: banik@iub.edu.bd
(For examples, see [2-4]). We know that point estimate vary from sample to sample. On the other hand, a $\mathrm{Cl}$ gives a range of $r$-values estimated from a given set of random sample that is likely to include $\rho$. Cls are calculated at a confidence level usually $95 \%$, but other Cls for example $90 \%, 99 \%$ can also be constructed for $\rho$. It is believed that Cls are more informative than the results of hypothesis tests since they provide a range of plausible values of $\rho$. Note that a $\mathrm{Cl}$ also includes a hypothesis testing by looking whether or not the interval contains zero. Thus, computing a $\mathrm{Cl}$ for $\rho$ is necessary and would be very useful in real life analysis.

The main purpose of this paper is to propose some new methods and compare with several existing methods in literature by means of a simulation study and recommend some useful Cls for the researchers as well practitioners. Hall et al. (1989) [5] compared the parametric bootstrap $\mathrm{Cl}$, the Fisher $\mathrm{z} \mathrm{Cl}$ and the Fisher bootstrap $\mathrm{Cl}$ to see which $\mathrm{Cl}$ performs the best based on their simulation studies. They used bivariate normal and bivariate lognormal distributions as data generating process. Their simulation results show that the Fisher $z$ $\mathrm{Cl}$ is performed better than the parametric bootstrap $\mathrm{Cl}$ and the Fisher bootstrap $\mathrm{Cl}$. Best of our knowledge, this is only simulation study available in literature. Thus, we believe that the findings of this paper will be a guideline for researchers and practitioners to select some good $\mathrm{Cls}$ for $\rho$. The coverage probability, mean width and standard deviation of the widths are considered as a performance criterion. The coverage probability may vary from distribution to distribution, but a good method should maintain small standard deviation. 
The paper is planned as follows. Existing and proposed methods for constructing $\mathrm{Cl}$ for $\rho$ are described in section 2. Simulation study design and simulation outcomes are discussed in section 3. Two real life examples are analyzed in section 4 to exemplify the findings of the paper. Finally, some concluding remarks are given in section 5 .

\section{CONFIDENCE INTERVAL (CI) FOR THE POPULATION CORRELATION COEFFICIENT $\rho$}

Suppose we are interested to find the linear relationship between two variables $\mathrm{X}$ and $\mathrm{Y}$. The population correlation coefficient between two variables $X$ and $Y$ is denoted by $\rho$ and defined as follows

$\rho=\frac{\operatorname{Cov}(\mathrm{X}, \mathrm{Y})}{\sqrt{\operatorname{Var}(\mathrm{X}) \operatorname{Var}(\mathrm{Y})}}=\frac{\sigma_{\mathrm{XY}}}{\sqrt{\sigma_{\mathrm{X}}^{2} \sigma_{\mathrm{Y}}^{2}}}$.

The corresponding sample correlation coefficient $r$ is defined as follows

$r=\frac{\hat{\sigma}_{X Y}}{\sqrt{\hat{\sigma}_{X}^{2} \hat{\sigma}_{Y}^{2}}}$.

It can be shown that $-1 \leq \rho \leq 1$. A value of 1 implies that a linear equation describes the relationship between $X$ and $Y$ perfectly, with all data points lying on a straight line for which $Y$ increases as $X$ increases. A value of -1 implies that all data points lie on a straight line for which $\mathrm{Y}$ decreases as $\mathrm{X}$ increases. A value of 0 implies that there is no linear association between $X$ and $\mathrm{Y}$. Note that Cls computed for the parameter may vary depending on the size of the sample, confidence level and assumptions on the distribution of $r$. It can also be true that for the same sample size and for the same confidence level, the results would differ depending on the method will be used to calculate $\mathrm{Cl}$. Several Cls for estimating $\rho$ are described below.

\subsection{The Classical $\mathrm{Cl}(\mathrm{CCl})$}

Suppose the sample correlation coefficient, $r$ is computed from a random sample of size $n$ and considers as a point estimate for $\rho$. For the first time, Student (1908) [6] studied the distribution of $r$ when $\rho$ is zero. Thus, a common test is that of whether or not a relationship exists between two variables $\mathrm{X}$ and $\mathrm{Y}$. Under the null hypothesis,

$t=r \sqrt{\frac{n-2}{1-r^{2}}}$

has student's $t$ distribution with $n-2$ degrees of freedom (df). Thus, the $(1-\alpha) 100 \% \mathrm{Cl}$ for $\rho$ is obtained as $r \pm t_{(n-2), \frac{\alpha}{2}} S E(r)$

where $S E(r)=\sqrt{\frac{1-r^{2}}{d f}}$ and $t_{(n-2), \frac{\alpha}{2}}$ is the upper $(\alpha / 2)$ percentile point of a Student's t distribution with (n-2) df.

\subsection{Fisher's Large Sample z Cl (FCl)}

Calculation $\mathrm{Cl}$ based on $r$ is complicated by the fact that the sampling distribution of $r$ is not normally distributed. The exact distribution of $r$ was given by Fisher (1915) [7] based on geometrical methods. Since the sampling distribution of Pearson's $r$ is not normally distributed, Pearson's $r$ is converted to Fisher's $z$ and $\mathrm{Cl}$ is computed using Fisher's $z$ transformation.

The steps in computing a $\mathrm{Cl}$ for $\rho$ are:

(i) The Fisher $z$ transformation transforms $r$ into the variable $z$ which is approximately normal for any value of $r$ as long as $n$ is large enough.

(ii) The values of Fisher's $z$ in $\mathrm{Cl}$ are then converted back to Pearson's r.

Thus, for large $n, \hat{\Psi}=\frac{1}{2} \log \left(\frac{1+r}{1-r}\right) \approx N\left(\Psi, \frac{1}{\sqrt{n-3}}\right)$, where $\Psi=\frac{1}{2} \log \left(\frac{1+\rho}{1-\rho}\right)$. Then the approximate $(1-\alpha) 100 \% \mathrm{Cl}$ for $\rho$ is obtained (after some steps) as

$\frac{e^{2 l}-1}{e^{2 l}+1} \leq \rho \leq \frac{e^{2 u}-1}{e^{2 u}+1}$

where $1=\hat{\Psi}-z_{\alpha / 2} \frac{1}{\sqrt{n-3}}$ and $u=\hat{\Psi}+z_{1-\alpha / 2} \frac{1}{\sqrt{n-3}}$ and $\hat{\Psi}$ is defined above.

\subsection{Gorsuch and Lehmann Cls}

Recently, to improve coverage probability for all $n$, Gorsuch and Lehmann (2010) [8] modified the classical $\mathrm{Cl}$ and the Fisher-z $\mathrm{Cl}$ and proposed the following four Cls for $\rho$ namely $\mathrm{Clr}, \mathrm{Clr}(\mathrm{t}), \mathrm{Clz}$ and $\mathrm{Clz}(\mathrm{t})$ based on different standard errors (SE) of $r$ :

Modified classical Cls for $\rho$ :

The $(1-\alpha) 100 \% \mathrm{Cl}$ for $\rho$, Clr

$r \pm 2 S E(r)$ 
The $(1-\alpha) 100 \% \mathrm{Cl}$ for $\rho, \operatorname{Clr}(\mathrm{t})$

$r \pm t_{(n-1), \frac{\alpha}{2}} S E(r)$

where $\quad r=\frac{\mathrm{e}^{2 \hat{\Psi}}-1}{\mathrm{e}^{2 \hat{\Psi}}+1}, \hat{\Psi}=\frac{1}{2} \log \left(\frac{1+\mathrm{r}}{1-\mathrm{r}}\right)$ and $\mathrm{SE}(\mathrm{r})=\frac{1-\mathrm{r}^{2}}{\sqrt{\mathrm{n}-1}}$ and $t_{(n-1), \frac{\alpha}{2}}$ is the upper $(\alpha / 2)$ th percentile point from the t-distribution with $(n-1)$ degrees of freedom.

Modified Fisher Cls for $\rho$ :

The $(1-\alpha) 100 \% \mathrm{Cl}$ for $\rho, \mathrm{Clz}$

$\frac{e^{2 l}-1}{e^{2 l}+1} \leq \rho \leq \frac{e^{2 u}-1}{e^{2 u}+1}$,

where $l=\hat{\Psi}-\frac{2}{\sqrt{n-3}}$ and $u=\hat{\Psi}+\frac{2}{\sqrt{n-3}}$.

The $(1-\alpha) 100 \% \mathrm{Cl}$ for $\rho, \mathrm{Clz}(\mathrm{t})$

$\frac{\mathrm{e}^{21}-1}{\mathrm{e}^{21}+1} \leq \rho \leq \frac{\mathrm{e}^{2 \mathrm{u}}-1}{\mathrm{e}^{2 \mathrm{u}}+1}$

where $\mathrm{l}=\hat{\Psi}-\mathrm{t}_{(\mathrm{n}-1), \alpha / 2} \frac{1}{\sqrt{\mathrm{n}-3}}$ and $\mathrm{u}=\hat{\Psi}+\mathrm{t}_{(\mathrm{n}-1), \alpha / 2} \frac{1}{\sqrt{\mathrm{n}-3}}$.

\subsection{Proposed $\mathrm{Cl}(\mathrm{SKCl})$}

It is known that $r=b_{1} \times \frac{S_{x}}{S_{y}}$, where $\mathrm{b}_{1}$ is the ordinary least squares (OLS) estimator of $\beta_{1}$ for the linear regression model $y_{t}=\beta_{0}+\beta_{1} x_{t}+e_{t}, \beta_{0}$ is the constant, $\beta_{1}$ is the regression coefficient of $y$ on $x$ and $e_{t} \sim N\left(0, \sigma^{2}\right)$.

The $(1-\alpha) 100 \% \mathrm{Cl}$ for $\rho$ is given as

$r \pm t_{(n-2), \frac{\alpha}{2}} S E(r)$

where $\quad S E(r)=\frac{S_{x}}{S_{y}} \times S E\left(b_{1}\right)=\frac{S_{x}}{S_{y}} \times \sqrt{\frac{M S E}{\sum_{i=1}^{n}\left(x_{i}-\bar{x}\right)^{2}}} \quad$ and $t_{(n-2), \frac{\alpha}{2}}$ is the upper $(\alpha / 2)$ th percentile point of the Student's t distribution with (n-2) df.

We know that $S_{x}^{2}=\frac{\sum_{i=1}^{n}\left(x_{i}-x\right)^{2}}{n-1}=\sqrt{(n-1) S_{x}}=\sqrt{\sum_{i=1}^{n}\left(x_{i}-\bar{x}\right)^{2}}$. Then $S E(r)$ becomes $S E(r)=\sqrt{\frac{M S E}{\sum_{i=1}^{n}\left(y_{i}-\bar{y}\right)^{2}}}$. Thus, the $(1-\alpha) 100 \%$ Cl for $\rho$ would be

$$
r \pm t_{(n-2), \frac{\alpha}{2}} \sqrt{\frac{M S E}{\sum_{i=1}^{n}\left(y_{i}-\bar{y}\right)^{2}}} .
$$

\subsection{Non Parametric Bootstrap Cl (NBoot)}

Bootstrap is a computer-based non-parametric approach (established first by Efron (1979)) [9]. No assumptions require about the primary population and can be used to a variety of circumstances. The precision of the bootstrap $\mathrm{Cl}$ depends on the number of bootstrap samples. The bootstrap procedure is described as follows: Let $x^{(*)}=x_{1}^{(*)}, x_{2}^{(*)}, \ldots, x_{n}^{(*)}$ and $y^{(*)}$ $=y_{1}^{(*)}, y_{2}^{(*)}, \ldots, y_{n}^{(*)}$, where $i^{\text {th }}$ random samples are denoted by $x^{(i)}$ and $y^{(i)}$ for $i=1,2, \ldots, B$ and $B$ is the number of bootstrap samples. Calculate $r^{*}$ for all $\mathrm{B}$ samples and order $r$ of each $\mathrm{B}$. It is defined as follows: $r_{1}^{*} \leq r_{2}^{*} \leq \cdots \leq r_{B}^{*}$. The lower and upper $(1-\alpha) 100 \%$ confidence limits for $\rho$ are respectively,

$$
L C L=r_{\left(\frac{\alpha}{2}\right) B}^{*} \text { and } U C L=r_{\left(1-\frac{\alpha}{2}\right) B}^{*} \text {. }
$$

\subsection{Parametric Bootstrap of Classical $\mathrm{Cl}$ (PBoot)}

The $(1-\alpha) 100 \% \mathrm{Cl}$ for $\rho$ is obtained as

$r \pm t_{(n-2), \frac{\alpha}{2}}^{*} S E(r)$,

where $S E(r)=\sqrt{\frac{1-r^{2}}{d f}}$ and $t_{(n-2), \frac{\alpha}{2}}^{*}$ is the $(\alpha / 2)^{\text {th }}$ sample quintiles of $t^{*}=r^{*} \sqrt{\frac{n-2}{1-r^{* 2}}}$.

\subsection{Parametric Bootstrap of Fisher $\mathrm{z} \mathrm{Cl}$ (FBoot)}

The $(1-\alpha) 100 \% \mathrm{Cl}$ for $\rho$ is obtained as

$$
\frac{e^{2 l^{*}}-1}{e^{2 l^{*}}+1} \leq \rho \leq \frac{e^{2 u^{*}}-1}{e^{2 u^{*}}+1}
$$

where $l^{*}=\hat{\Psi}-Z_{\alpha / 2}^{*} \frac{1}{\sqrt{n-3}}$ and $u^{*}=\hat{\Psi}+Z_{\alpha / 2}^{*} \frac{1}{\sqrt{n-3}}, Z_{\alpha / 2}^{*}$ is the $(\alpha / 2)^{\text {th }}$ sample quintiles of $\hat{\Psi}^{*} / \frac{1}{\sqrt{n-3}}$, where $\hat{\Psi}^{*}$ is defined by $\frac{1}{2} \log \left(\frac{1+r^{*}}{1-r^{*}}\right)$ and $r^{*}$ is the correlation of all B samples.

\subsection{Parametric Bootstrap of Proposed Method Cl (SKBoot)}

The $(1-\alpha) 100 \% \mathrm{Cl}$ for $\rho$ 
$r \pm t_{(n-2), \frac{\alpha}{2}}^{*} \sqrt{\frac{M S E}{\sum_{i=1}^{n}\left(y_{i}-\overline{\bar{y}}\right)^{2}}}$,

where $\overline{\bar{y}}=\frac{1}{B} \sum_{i=1}^{B} \bar{y}_{i}^{*}$ and $t_{(n-2), \frac{\alpha}{2}}^{*}$ is the $(\alpha / 2)^{\text {th }}$ sample quintiles of $t^{*}=r^{*} \sqrt{\frac{n-2}{1-r^{* 2}}}$.

\subsection{Bootstrap Bias Corrected Acceleration (BCA) Cl}

This method is introduced by Efron and Tibshirani (1993) [10]. BCA Cl for $\rho$ is defined as

$L C L=r^{\left(\alpha_{1}\right)}$ and $U C L=r^{\left(\alpha_{2}\right)}$

where $r^{\left(\alpha_{1}\right)}$ and $r^{\left(\alpha_{2}\right)}$ are the $\left(\alpha_{1} 100\right) \%$ and $\left(\alpha_{2} 100\right) \%$ percentiles of the distribution of $r_{i}^{*}$,

$\alpha_{1}=\phi\left[\hat{\mathrm{z}}_{0}+\frac{\hat{\mathrm{z}}_{0}+\mathrm{z}^{(\alpha)}}{1-\hat{\mathrm{a}}\left\{\hat{\mathrm{z}}_{0}+\mathrm{z}^{(\alpha)}\right\}}\right]$ and $\alpha_{2}=\phi\left[\hat{\mathrm{z}}_{0}+\frac{\hat{\mathrm{z}}_{0}+\mathrm{z}^{(1-\alpha)}}{1-\hat{\mathrm{a}}\left\{\hat{\mathrm{z}}_{0}+\mathrm{z}^{(1-\alpha)}\right\}}\right], \phi($. is the standard normal cumulative distribution function, $z^{(1-\alpha)}$ is the $100^{*}(1-\alpha)^{\text {th }}$ percentile of the $Z$ distribution, bias correction $\hat{\mathrm{z}}_{0}=\phi^{-1}\left(\frac{\#\left(\mathrm{r}_{\mathrm{i}}^{*}<\mathrm{r}\right)}{\mathrm{B}}\right), \phi^{-1}($.) is the inverse function of cumulative distribution function of the $\mathrm{Z}$ distribution, acceleration factor $a=\sum_{i=1}^{n}\left(r-r_{-i}\right)^{3} / 6\left[\sum_{i=1}^{n}\left(r-r_{-i}\right)^{2}\right]^{1.5}, r$ is the correlation between $x$ and $y$ and $r_{i}$ is the correlation between $x$ and $y$ of $(n-1)$ observations without the $i^{\text {th }}$ observation.

\section{SIMULATION STUDY}

The goal of this paper is to evaluate the performance of $\mathrm{Cl}$ estimators for $\rho$, which have been discussed in section 2. Since a theoretical comparison among the estimators is not possible, a simulation study has been conducted in this section.

\subsection{Simulation Design}

The most common $95 \% \mathrm{Cl}$ is computed and assumed random sample sizes $n=10,20,30,50$ and 100 and $\rho_{1}$ (correlation between two variables is used to compute covariance matrix of the considered distributions) $=0.7,0.8$ and 0.9 . Random samples were generated from the following distributions:

(a) Bivariate normal with $\mu_{1}=15, \mu_{2}=20$ and $\sigma_{1}=10$, $\sigma_{2}=4$

(b) Bivariate log normal with $\mu_{1}=15, \mu_{2}=20$ and $\sigma_{1}=10, \sigma_{2}=4$ (c) Bivariate chi-square with df 1 and 3 respectively

We have considered 2500 replications for our simulation experiments and 1500 bootstrap samples for each selected random sample. To evaluate the performance of selected Cls, following criterions are chosen: (i) coverage probability (lower, cover and upper) (ii) mean width (iii) standard deviation (SD) of the width. It is well-known that the coverage probability will be precise or near to $(1-\alpha),(\alpha$ is the level of significance) if random samples come from the symmetric distribution. Thus, it is believable that coverage probability is a constructive measure for assessing $\mathrm{Cl}$. Another important measure is the width of $\mathrm{Cl}$. A shorter width provides a better $\mathrm{Cl}$. It is also true that when coverage probability is equal for two or more $\mathrm{Cls}$, in that case a smaller width indicates that the technique is suitable for the particular sample.

\subsection{Results discussion}

We have reported evaluation measures of the selected Cls in Tables $\mathbf{1}$ to $\mathbf{5}$ for selected $\mathrm{n}$ and $\rho_{1}$ when random samples are generated from bivariate normal, bivariate lognormal and bivariate chi-square distributions. For a visual expression, simulation results are presented graphically in Figures $\mathbf{1}$ to $\mathbf{5}$.

For various values of $n$ and $\rho_{1}$, we have presented simulation results when data are generated from the bivariate normal distribution in Tables $\mathbf{1}$ to $\mathbf{3}$. Figure $\mathbf{1}$ shows coverage probablity for various values of $n$ and $\rho_{1}=0.7$. It is clear that as $n$ increases, coverage probabilities also increases. It is observed that the coverage probability of BCA, SKBoot, $\mathrm{FCl}$ and $\mathrm{Clz}$ are close to nominal level 0.95 as compare to others. Overall BCA interval is performing the best in the sense of correct coverage probability and smaller width. On the contrary, estimators $\mathrm{Clr}$ and $\mathrm{Clr}(\mathrm{t})$ show poor performances in terms of coverage probability compare to others.

To understand the effect of $\rho_{1}$ on Cls, coverage probabilities are presented in Figure 2 for $\rho_{1}=0.7,0.8$ and 0.9 for $n=30$. It is found that as $\rho_{1}$ increases cover rates changes. For various selected values of $\rho_{1}, \mathrm{BCA}$, SKBoot, PBoot, $\mathrm{Clz}$ and $\mathrm{FCl}$ have coverage probabilities around 0.95 . Among them $\mathrm{BCA}$ is the best in the sense of high coverage probability as compare to others.

To see the performance in the sense of smaller width, we have presented mean widths w.r.t sample 
Table 1: $95 \%$ Confidence Interval when Data Generated from Bivariate Normal for $\rho_{1}=0.7$

\begin{tabular}{|c|c|c|c|c|c|c|c|c|c|c|c|c|}
\hline Critria & $\mathrm{CCl}$ & $\mathrm{FCl}$ & Clr & $\operatorname{CIr}(\mathrm{t})$ & $\mathrm{Clz}$ & $\mathrm{Clz}(\mathrm{t})$ & SKCl & NBoot & PBoot & FBoot & SKBoot & BCA \\
\hline \multicolumn{13}{|c|}{$n=10$} \\
\hline B Rate & 0.0460 & 0.0350 & 0.0010 & 0.0010 & 0.0200 & 0.0100 & 0.0010 & 0.0000 & 0.0010 & 0.0040 & 0.0310 & 0.0260 \\
\hline C Rate & 0.8310 & 0.9250 & 0.8010 & 0.7990 & 0.9340 & 0.8110 & 0.8220 & 0.8220 & 0.8010 & 0.8680 & 0.9310 & 0.9530 \\
\hline O Rate & 0.1230 & 0.0400 & 0.1980 & 0.2000 & 0.0460 & 0.1790 & 0.1770 & 0.1780 & 0.1980 & 0.1280 & 0.0380 & 0.0210 \\
\hline MW & 1.5254 & 1.1573 & 1.1759 & 1.3300 & 1.2111 & 1.7100 & 1.5230 & 1.2609 & 1.1228 & 1.1328 & 1.1348 & 0.7828 \\
\hline SDW & 0.1347 & 0.1331 & 0.1876 & 0.2122 & 0.0000 & 0.0000 & 0.1250 & 0.0254 & 0.1345 & 0.1324 & 0.1302 & 0.3149 \\
\hline \multicolumn{13}{|c|}{$n=20$} \\
\hline B Rate & 0.0060 & 0.0380 & 0.0040 & 0.0040 & 0.0240 & 0.0030 & 0.0020 & 0.0040 & 0.0280 & 0.0060 & 0.0260 & 0.0260 \\
\hline C Rate & 0.9410 & 0.9310 & 0.8400 & 0.8420 & 0.9410 & 0.8830 & 0.8580 & 0.8520 & 0.9200 & 0.8820 & 0.9320 & 0.9540 \\
\hline O Rate & 0.0530 & 0.0310 & 0.1560 & 0.1540 & 0.0350 & 0.1140 & 0.1400 & 0.1440 & 0.0520 & 0.1120 & 0.0420 & 0.0200 \\
\hline MW & 0.9561 & 0.9264 & 0.8571 & 0.8970 & 0.9701 & 1.0153 & 0.9517 & 0.8861 & 0.9846 & 0.8649 & 0.9028 & 0.5366 \\
\hline SDW & 0.0456 & 0.0637 & 0.0771 & 0.0807 & 0.0000 & 0.0000 & 0.0411 & 0.0199 & 0.0469 & 0.0643 & 0.0409 & 0.2369 \\
\hline \multicolumn{13}{|c|}{$n=30$} \\
\hline B Rate & 0.0010 & 0.0140 & 0.0390 & 0.0090 & 0.0340 & 0.0140 & 0.0030 & 0.0070 & 0.0060 & 0.0060 & 0.0130 & 0.0240 \\
\hline C Rate & 0.9720 & 0.9380 & 0.8450 & 0.8670 & 0.9440 & 0.9010 & 0.9030 & 0.8830 & 0.9320 & 0.9290 & 0.9370 & 0.9560 \\
\hline O Rate & 0.0270 & 0.0480 & 0.1160 & 0.1240 & 0.0220 & 0.0850 & 0.0940 & 0.1100 & 0.0620 & 0.0650 & 0.0500 & 0.0200 \\
\hline MW & 0.7552 & 0.6903 & 0.7075 & 0.7235 & 0.7998 & 0.7872 & 0.7457 & 0.7213 & 0.7986 & 0.7732 & 0.7475 & 0.4465 \\
\hline SDW & 0.0254 & 0.0398 & 0.0457 & 0.0468 & 0.0000 & 0.0000 & 0.0242 & 0.0168 & 0.0251 & 0.0384 & 0.0240 & 0.1938 \\
\hline \multicolumn{13}{|c|}{$n=50$} \\
\hline B Rate & 0.0000 & 0.0210 & 0.0120 & 0.0180 & 0.0270 & 0.0060 & 0.0110 & 0.0090 & 0.0330 & 0.0400 & 0.0140 & 0.0220 \\
\hline C Rate & 0.9950 & 0.9390 & 0.8570 & 0.8700 & 0.9480 & 0.9500 & 0.9270 & 0.9220 & 0.9330 & 0.9305 & 0.9380 & 0.9580 \\
\hline O Rate & 0.0050 & 0.0300 & 0.1310 & 0.1120 & 0.0250 & 0.0440 & 0.0620 & 0.0690 & 0.0340 & 0.0295 & 0.0480 & 0.0200 \\
\hline MW & 0.5702 & 0.5489 & 0.5517 & 0.5544 & 0.5335 & 0.5863 & 0.5671 & 0.5573 & 0.5943 & 0.5826 & 0.5594 & 0.3318 \\
\hline SDW & 0.0129 & 0.0222 & 0.0246 & 0.0245 & 0.0000 & 0.0000 & 0.0128 & 0.0128 & 0.0133 & 0.0229 & 0.0132 & 0.1593 \\
\hline \multicolumn{13}{|c|}{$n=100$} \\
\hline B Rate & 0.0000 & 0.0220 & 0.0200 & 0.0340 & 0.0130 & 0.0180 & 0.0120 & 0.0140 & 0.0150 & 0.0150 & 0.0220 & 0.0280 \\
\hline C Rate & 1.0000 & 0.9480 & 0.8580 & 0.8700 & 0.9690 & 0.9620 & 0.9410 & 0.9240 & 0.9470 & 0.9340 & 0.9450 & 0.9620 \\
\hline O Rate & 0.0000 & 0.0200 & 0.1220 & 0.0960 & 0.0180 & 0.0200 & 0.0470 & 0.0620 & 0.0380 & 0.0510 & 0.0330 & 0.0100 \\
\hline MW & 0.4966 & 0.4148 & 0.3835 & 0.3904 & 0.4061 & 0.4029 & 0.3765 & 0.3930 & 0.4157 & 0.4101 & 0.3846 & 0.2108 \\
\hline SDW & 0.0050 & 0.0093 & 0.0098 & 0.0098 & 0.0000 & 0.0000 & 0.0051 & 0.0093 & 0.0049 & 0.0090 & 0.0052 & 0.1226 \\
\hline
\end{tabular}

Table 2: $95 \%$ Confidence Interval when Data Generated from Bivariate Normal for $\rho_{1}=0.8$

\begin{tabular}{|c|c|c|c|c|c|c|c|c|c|c|c|c|}
\hline Critria & CCI & FCl & Clr & Clr(t) & Clz & Clz(t) & SKCl & NBoot & PBoot & FBoot & SKBoot & BCA \\
\hline \hline \multicolumn{10}{|c|}{$\mathrm{n}=10$} \\
\hline B Rate & 0.0400 & 0.0350 & 0.0010 & 0.0010 & 0.0240 & 0.0210 & 0.0000 & 0.0010 & 0.0210 & 0.0010 & 0.0320 & 0.0270 \\
\hline C Rate & 0.8240 & 0.9220 & 0.7530 & 0.7510 & 0.9310 & 0.7430 & 0.7600 & 0.7920 & 0.8005 & 0.7600 & 0.9300 & 0.9520 \\
\hline O Rate & 0.1360 & 0.0430 & 0.2460 & 0.2480 & 0.0450 & 0.2360 & 0.2400 & 0.2070 & 0.1785 & 0.2390 & 0.0380 & 0.0210 \\
\hline MW & 1.5222 & 1.5141 & 1.1721 & 1.3257 & 1.5119 & 1.7100 & 1.5212 & 1.2607 & 1.4381 & 1.1786 & 1.1163 & 0.7693 \\
\hline SDW & 0.1427 & 0.1411 & 0.1964 & 0.2221 & 0.0000 & 0.0000 & 0.1376 & 0.0423 & 0.1348 & 0.1380 & 0.1252 & 0.3440 \\
\hline
\end{tabular}


(Table 2). Continued.

\begin{tabular}{|c|c|c|c|c|c|c|c|c|c|c|c|c|}
\hline Critria & $\mathrm{CCl}$ & $\mathrm{FCl}$ & Clr & $\operatorname{CIr}(t)$ & $\mathrm{Clz}$ & $\mathrm{Clz}(\mathrm{t})$ & SKCl & NBoot & PBoot & FBoot & SKBoot & BCA \\
\hline \multicolumn{13}{|c|}{$\mathrm{n}=\mathbf{2 0}$} \\
\hline B Rate & 0.0040 & 0.0370 & 0.0040 & 0.0040 & 0.0295 & 0.0120 & 0.0060 & 0.0030 & 0.0280 & 0.0020 & 0.0370 & 0.0210 \\
\hline C Rate & 0.9360 & 0.9300 & 0.8330 & 0.8350 & 0.9405 & 0.8630 & 0.8460 & 0.8670 & 0.9190 & 0.8270 & 0.9310 & 0.9520 \\
\hline O Rate & 0.0600 & 0.0310 & 0.1630 & 0.1610 & 0.0300 & 0.1250 & 0.1480 & 0.1300 & 0.0530 & 0.1710 & 0.0320 & 0.0290 \\
\hline MW & 1.0565 & 1.0169 & 0.8577 & 0.8976 & 0.9701 & 1.0153 & 0.9473 & 0.8876 & 1.0196 & 0.8917 & 0.8864 & 0.5563 \\
\hline SDW & 0.0435 & 0.0610 & 0.0742 & 0.0777 & 0.0000 & 0.0000 & 0.0421 & 0.0339 & 0.0463 & 0.0663 & 0.0394 & 0.2370 \\
\hline \multicolumn{13}{|c|}{$n=30$} \\
\hline B Rate & 0.0010 & 0.0120 & 0.0380 & 0.0320 & 0.0350 & 0.0040 & 0.0030 & 0.0050 & 0.0060 & 0.0040 & 0.0120 & 0.0240 \\
\hline C Rate & 0.9820 & 0.9350 & 0.8360 & 0.8580 & 0.9410 & 0.9140 & 0.9010 & 0.9160 & 0.9310 & 0.9000 & 0.9330 & 0.9520 \\
\hline O Rate & 0.0170 & 0.0430 & 0.1260 & 0.1100 & 0.0240 & 0.0820 & 0.0960 & 0.0790 & 0.0630 & 0.0960 & 0.0450 & 0.0240 \\
\hline MW & 0.7544 & 0.7890 & 0.7060 & 0.7219 & 0.7698 & 0.7872 & 0.7244 & 0.7205 & 0.7132 & 0.7337 & 0.7055 & 0.4505 \\
\hline SDW & 0.0235 & 0.0369 & 0.0426 & 0.0436 & 0.0000 & 0.0000 & 0.0255 & 0.0284 & 0.0222 & 0.0364 & 0.0265 & 0.1971 \\
\hline \multicolumn{13}{|c|}{$n=50$} \\
\hline B Rate & 0.0000 & 0.0220 & 0.0080 & 0.0080 & 0.0230 & 0.0040 & 0.0090 & 0.0060 & 0.0340 & 0.0120 & 0.0140 & 0.0220 \\
\hline C Rate & 0.9980 & 0.9370 & 0.8630 & 0.8700 & 0.9440 & 0.9350 & 0.9220 & 0.9240 & 0.9320 & 0.9330 & 0.9350 & 0.9530 \\
\hline O Rate & 0.0020 & 0.0310 & 0.1290 & 0.1220 & 0.0330 & 0.0610 & 0.0690 & 0.0700 & 0.0340 & 0.0550 & 0.0510 & 0.0250 \\
\hline MW & 0.6702 & 0.5989 & 0.5018 & 0.5544 & 0.5835 & 0.5863 & 0.5696 & 0.5571 & 0.6204 & 0.5383 & 0.5297 & 0.3141 \\
\hline SDW & 0.0127 & 0.0218 & 0.0240 & 0.0241 & 0.0000 & 0.0000 & 0.0127 & 0.0226 & 0.0138 & 0.0200 & 0.0125 & 0.1679 \\
\hline \multicolumn{13}{|c|}{$n=100$} \\
\hline B Rate & 0.0000 & 0.0230 & 0.0110 & 0.0090 & 0.0160 & 0.0070 & 0.0100 & 0.0120 & 0.0150 & 0.0050 & 0.0210 & 0.0280 \\
\hline C Rate & 1.0000 & 0.9470 & 0.8670 & 0.8790 & 0.9660 & 0.9530 & 0.9330 & 0.9400 & 0.9450 & 0.9420 & 0.9430 & 0.9610 \\
\hline O Rate & 0.0000 & 0.0210 & 0.1220 & 0.1120 & 0.0180 & 0.0400 & 0.0570 & 0.0480 & 0.0300 & 0.0530 & 0.0360 & 0.0110 \\
\hline MW & 0.4956 & 0.4330 & 0.3716 & 0.3885 & 0.4061 & 0.4029 & 0.3956 & 0.3936 & 0.4006 & 0.3934 & 0.3814 & 0.1825 \\
\hline SDW & 0.0056 & 0.0103 & 0.0109 & 0.0109 & 0.0000 & 0.0000 & 0.0059 & 0.0166 & 0.0068 & 0.0103 & 0.0057 & 0.1284 \\
\hline
\end{tabular}

Table 3: $95 \%$ Confidence Interval when Data Generated from Bivariate Normal for $\rho_{1}=0.9$

\begin{tabular}{|c|c|c|c|c|c|c|c|c|c|c|c|c|c|}
\hline Critria & CCI & FCl & Clr & Clr(t) & Clz & Clz(t) & SKCl & NBoot & PBoot & FBoot & SKBoot & BCA \\
\hline \hline \multicolumn{10}{|c|}{$\mathbf{n = 1 0}$} \\
\hline B Rate & 0.0410 & 0.0360 & 0.0000 & 0.0000 & 0.0240 & 0.0200 & 0.0020 & 0.0000 & 0.0210 & 0.0000 & 0.0320 & 0.0245 \\
\hline C Rate & 0.8100 & 0.9210 & 0.6940 & 0.6890 & 0.9310 & 0.7100 & 0.7300 & 0.7430 & 0.8005 & 0.6790 & 0.9300 & 0.9500 \\
\hline O Rate & 0.1490 & 0.0430 & 0.3060 & 0.3110 & 0.0450 & 0.2700 & 0.2680 & 0.2570 & 0.1785 & 0.3210 & 0.0380 & 0.0255 \\
\hline MW & 1.5169 & 1.4989 & 1.1641 & 1.3167 & 1.5119 & 1.7100 & 1.5141 & 1.5667 & 1.5166 & 1.1743 & 1.1691 & 0.7626 \\
\hline SDW & 0.1433 & 0.1418 & 0.1984 & 0.2245 & 0.0000 & 0.0000 & 0.1416 & 0.1480 & 0.1404 & 0.1354 & 0.1414 & 0.3425 \\
\hline & & & & & & & & \\
\hline B Rate & 0.0020 & 0.0310 & 0.0020 & 0.0020 & 0.0295 & 0.0300 & 0.0040 & 0.0020 & 0.0280 & 0.0020 & 0.0370 & 0.0270 \\
\hline C Rate & 0.9430 & 0.9280 & 0.8110 & 0.8120 & 0.9405 & 0.8530 & 0.8360 & 0.8590 & 0.9190 & 0.8390 & 0.9310 & 0.9510 \\
\hline O Rate & 0.0550 & 0.0310 & 0.1870 & 0.1860 & 0.0300 & 0.1170 & 0.1600 & 0.1390 & 0.0530 & 0.1590 & 0.0320 & 0.0220 \\
\hline MW & 1.0855 & 1.0356 & 0.8561 & 0.8959 & 0.9701 & 1.0153 & 0.9529 & 0.8864 & 0.9906 & 0.8789 & 0.8417 & 0.5534 \\
\hline SDW & 0.0454 & 0.0633 & 0.0765 & 0.0801 & 0.0000 & 0.0000 & 0.0477 & 0.0340 & 0.0471 & 0.0652 & 0.0477 & 0.2320 \\
\hline
\end{tabular}


(Table 3). Continued.

\begin{tabular}{|c|c|c|c|c|c|c|c|c|c|c|c|c|}
\hline Critria & $\mathrm{CCl}$ & $\mathrm{FCl}$ & Clr & $C \operatorname{lr}(t)$ & $\mathrm{Clz}$ & $\mathrm{Clz}(\mathrm{t})$ & SKCl & NBoot & PBoot & FBoot & SKBoot & BCA \\
\hline \multicolumn{13}{|c|}{$n=30$} \\
\hline B Rate & 0.0010 & 0.0140 & 0.0340 & 0.0050 & 0.0350 & 0.0050 & 0.0060 & 0.0050 & 0.0060 & 0.0050 & 0.0120 & 0.0200 \\
\hline C Rate & 0.9640 & 0.9330 & 0.8280 & 0.8440 & 0.9410 & 0.8940 & 0.8660 & 0.9100 & 0.9310 & 0.8630 & 0.9330 & 0.9510 \\
\hline O Rate & 0.0350 & 0.0430 & 0.1380 & 0.1510 & 0.0240 & 0.1010 & 0.1280 & 0.0850 & 0.0630 & 0.1320 & 0.0450 & 0.0290 \\
\hline MW & 0.8522 & 0.8856 & 0.7021 & 0.7180 & 0.7698 & 0.7872 & 0.7519 & 0.7199 & 0.7690 & 0.7132 & 0.7048 & 0.4134 \\
\hline SDW & 0.0277 & 0.0432 & 0.0496 & 0.0507 & 0.0000 & 0.0000 & 0.0280 & 0.0282 & 0.0272 & 0.0415 & 0.0296 & 0.2080 \\
\hline \multicolumn{13}{|c|}{$\mathrm{n}=50$} \\
\hline B Rate & 0.0000 & 0.0220 & 0.0050 & 0.0290 & 0.0230 & 0.0020 & 0.0040 & 0.0130 & 0.0340 & 0.0130 & 0.0140 & 0.0230 \\
\hline C Rate & 0.9930 & 0.9360 & 0.8380 & 0.8650 & 0.9440 & 0.9430 & 0.9040 & 0.9170 & 0.9320 & 0.9030 & 0.9350 & 0.9520 \\
\hline O Rate & 0.0070 & 0.0320 & 0.1570 & 0.1060 & 0.0330 & 0.0550 & 0.0920 & 0.0700 & 0.0340 & 0.0840 & 0.0510 & 0.0250 \\
\hline MW & 0.6688 & 0.6364 & 0.5091 & 0.5517 & 0.5835 & 0.5863 & 0.5603 & 0.5566 & 0.5921 & 0.5311 & 0.5153 & 0.3099 \\
\hline SDW & 0.0143 & 0.0244 & 0.0267 & 0.0269 & 0.0000 & 0.0000 & 0.0139 & 0.0232 & 0.0146 & 0.0248 & 0.0126 & 0.1690 \\
\hline \multicolumn{13}{|c|}{$n=100$} \\
\hline B Rate & 0.0000 & 0.0230 & 0.0090 & 0.0060 & 0.0160 & 0.0170 & 0.0090 & 0.0040 & 0.0150 & 0.0040 & 0.0210 & 0.0200 \\
\hline C Rate & 1.0000 & 0.9440 & 0.8560 & 0.8760 & 0.9660 & 0.9500 & 0.9290 & 0.9310 & 0.9450 & 0.9260 & 0.9430 & 0.9590 \\
\hline O Rate & 0.0000 & 0.0230 & 0.1350 & 0.1180 & 0.0180 & 0.0330 & 0.0620 & 0.0650 & 0.0300 & 0.0700 & 0.0360 & 0.0310 \\
\hline MW & 0.4948 & 0.4514 & 0.3819 & 0.3868 & 0.4061 & 0.4029 & 0.3901 & 0.3929 & 0.4225 & 0.3988 & 0.3823 & 0.1693 \\
\hline SDW & 0.0062 & 0.0114 & 0.0121 & 0.0120 & 0.0000 & 0.0000 & 0.0060 & 0.0162 & 0.0061 & 0.0109 & 0.0062 & 0.1241 \\
\hline
\end{tabular}

Table 4: $95 \%$ Confidence Interval when Data Generated from Bivariate Lognormal for $\rho_{1}=0.7$

\begin{tabular}{|c|c|c|c|c|c|c|c|c|c|c|c|c|}
\hline Critria & CCI & $\mathrm{FCl}$ & Clr & $\operatorname{Clr}(\mathrm{t})$ & Clz & $\mathrm{Clz}(\mathrm{t})$ & SKCl & NBoot & PBoot & FBoot & SKBoot & BCA \\
\hline \multicolumn{13}{|c|}{$n=10$} \\
\hline B Rate & 0.0050 & 0.0200 & 0.0050 & 0.0020 & 0.0240 & 0.0000 & 0.0000 & 0.0140 & 0.0250 & 0.0000 & 0.0200 & 0.0140 \\
\hline C Rate & 0.8570 & 0.9420 & 0.8600 & 0.8680 & 0.9410 & 0.8870 & 0.8960 & 0.8990 & 0.9420 & 0.9010 & 0.9450 & 0.9520 \\
\hline O Rate & 0.1380 & 0.0380 & 0.1350 & 0.1280 & 0.0350 & 0.1130 & 0.1040 & 0.0870 & 0.0330 & 0.0990 & 0.0350 & 0.0340 \\
\hline MW & 1.5043 & 1.7386 & 1.1689 & 1.3221 & 1.5119 & 1.7100 & 1.4959 & 1.2348 & 1.7269 & 1.1920 & 1.4878 & 0.8093 \\
\hline SDW & 0.2607 & 0.2450 & 0.2910 & 0.3291 & 0.0000 & 0.0000 & 0.3761 & 0.0666 & 0.2993 & 0.3204 & 0.4106 & 0.3601 \\
\hline \multicolumn{13}{|c|}{$\mathrm{n}=\mathbf{2 0}$} \\
\hline B Rate & 0,0000 & 0.0210 & 0.0000 & 0.0000 & 0.0200 & 0.0010 & 0.0000 & 0.0020 & 0.0120 & 0.0050 & 0.0240 & 0.0230 \\
\hline C Rate & 0.8880 & 0.9440 & 0.8640 & 0.8690 & 0.9430 & 0.9250 & 0.9160 & 0.9350 & 0.9470 & 0.9370 & 0.9460 & 0.9530 \\
\hline O Rate & 0.1120 & 0.0350 & 0.1360 & 0.1310 & 0.0370 & 0.0740 & 0.0840 & 0.0630 & 0.0410 & 0.0580 & 0.0400 & 0.0240 \\
\hline MW & 0.9428 & 1.1223 & 0.8454 & 0.8847 & 0.9701 & 1.0153 & 0.9370 & 0.8776 & 1.1297 & 0.8241 & 0.9239 & 0.6313 \\
\hline SDW & 0.1215 & 0.1471 & 0.1626 & 0.1702 & 0.0000 & 0.0000 & 0.1587 & 0.0476 & 0.1456 & 0.1657 & 0.2240 & 0.2930 \\
\hline \multicolumn{13}{|c|}{$n=30$} \\
\hline B Rate & 0.0000 & 0.0230 & 0.0000 & 0.0000 & 0.0200 & 0.0000 & 0.0000 & 0.0050 & 0.0210 & 0,0210 & 0.0200 & 0.0020 \\
\hline C Rate & 0.9420 & 0.9450 & 0.9040 & 0.9040 & 0.9520 & 0.9300 & 0.9190 & 0.9360 & 0.9490 & 0.9410 & 0.9480 & 0.9560 \\
\hline O Rate & 0.0580 & 0.0320 & 0.0960 & 0.0960 & 0.0380 & 0.0700 & 0.0810 & 0.0590 & 0.0300 & 0.0380 & 0.0320 & 0.0420 \\
\hline MW & 0.7544 & 0.8914 & 0.7100 & 0.7261 & 0.7698 & 0.7872 & 0.7147 & 0.7269 & 0.7603 & 0.6407 & 0.7129 & 0.3786 \\
\hline SDW & 0.0626 & 0.0850 & 0.0922 & 0.0943 & 0.0000 & 0.0000 & 0.0997 & 0.0489 & 0.0630 & 0.0956 & 0.0958 & 0.2310 \\
\hline
\end{tabular}


(Table 4). Continued.

\begin{tabular}{|c|c|c|c|c|c|c|c|c|c|c|c|c|}
\hline Critria & $\mathrm{CCl}$ & $\mathrm{FCl}$ & Clr & $\operatorname{Clr}(t)$ & $\mathrm{Clz}$ & $\mathrm{Clz}(\mathrm{t})$ & SKCl & NBoot & PBoot & FBoot & SKBoot & BCA \\
\hline \multicolumn{13}{|c|}{$n=50$} \\
\hline B.Rate & 0.0000 & 0.0210 & 0.0000 & 0.0000 & 0.0200 & 0.0000 & 0.0000 & 0.0010 & 0.0030 & 0.0310 & 0.0200 & 0.0190 \\
\hline C Rate & 0.9500 & 0.9480 & 0.9090 & 0.9150 & 0.9520 & 0.9320 & 0.9260 & 0.9370 & 0.9470 & 0.9460 & 0.9490 & 0.9570 \\
\hline O Rate & 0.0500 & 0.0410 & 0.0910 & 0.0850 & 0.0280 & 0.0680 & 0.0740 & 0.0620 & 0.0500 & 0.0230 & 0.0310 & 0.0240 \\
\hline MW & 0.5680 & 0.6374 & 0.5507 & 0.5533 & 0.5835 & 0.5863 & 0.5485 & 0.5683 & 0.6237 & 0.6406 & 0.5525 & 0.2730 \\
\hline SDW & 0.0458 & 0.0640 & 0.0675 & 0.0678 & 0.0000 & 0.0000 & 0.0545 & 0.0459 & 0.0503 & 0.0974 & 0.0531 & 0.2409 \\
\hline \multicolumn{13}{|c|}{$n=100$} \\
\hline B Rate & 0.0000 & 0.0210 & 0.0000 & 0.0000 & 0.0250 & 0.0000 & 0.0000 & 0.0150 & 0.0240 & 0.0310 & 0.0210 & 0.0200 \\
\hline C Rate & 0.9840 & 0.9490 & 0.9310 & 0.9310 & 0.9530 & 0.9500 & 0.9360 & 0.9510 & 0.9510 & 0.9460 & 0.9500 & 0.9580 \\
\hline O Rate & 0.0160 & 0.0300 & 0.0690 & 0.0690 & 0.0220 & 0.0500 & 0.0340 & 0.0340 & 0.0250 & 0.0230 & 0.0290 & 0.0220 \\
\hline MW & 0.3971 & 0.4667 & 0.3956 & 0.3925 & 0.4061 & 0.4029 & 0.3188 & 0.3938 & 0.4595 & 0.4142 & 0.3106 & 0.2489 \\
\hline SDW & 0.0212 & 0.0294 & 0.0304 & 0.0302 & 0.0000 & 0.0000 & 0.0271 & 0.0435 & 0.0245 & 0.0374 & 0.0330 & 0.2614 \\
\hline
\end{tabular}

Table 5: $95 \%$ Confidence Interval when Data Generated from Bivariate Chi-Square for $\rho_{1}=0.7$

\begin{tabular}{|c|c|c|c|c|c|c|c|c|c|c|c|c|}
\hline Critria & $\mathrm{CCl}$ & $\mathrm{FCl}$ & Clr & $\operatorname{Clr}(t)$ & $\mathrm{Clz}$ & $\mathrm{Clz}(\mathrm{t})$ & SKCl & NBoot & PBoot & FBoot & SKBoot & $\mathrm{BCA}$ \\
\hline \multicolumn{13}{|c|}{$n=10$} \\
\hline B Rate & 0.0680 & 0.0240 & 0.0620 & 0.0500 & 0.0220 & 0.0100 & 0.0240 & 0.0500 & 0.0220 & 0.0500 & 0.0420 & 0.0160 \\
\hline C Rate & 0.8200 & 0.9420 & 0.8300 & 0.8760 & 0.9460 & 0.9480 & 0.9420 & 0.9280 & 0.9440 & 0.9260 & 0.9340 & 0.9480 \\
\hline O Rate & 0.1120 & 0.0340 & 0.1080 & 0.0740 & 0.0320 & 0.0420 & 0.0340 & 0.0220 & 0.0340 & 0.0240 & 0.0240 & 0.0360 \\
\hline MW & 1.5252 & 1.6573 & 1.1749 & 1.3289 & 1.5119 & 1.7100 & 1.3845 & 1.2485 & 1.5990 & 1.1904 & 1.3988 & 0.7659 \\
\hline SDW & 0.1292 & 0.1275 & 0.1819 & 0.2058 & 0.0000 & 0.0000 & 0.1104 & 0.0459 & 0.1355 & 0.1306 & 0.1254 & 0.3013 \\
\hline \multicolumn{13}{|c|}{$n=20$} \\
\hline B Rate & 0.0100 & 0.0200 & 0.0380 & 0.0360 & 0.0180 & 0.0200 & 0.0200 & 0.0120 & 0.0210 & 0.0180 & 0.0260 & 0.0280 \\
\hline C Rate & 0.9620 & 0.9440 & 0.9160 & 0.9220 & 0.9480 & 0.9540 & 0.9440 & 0.9380 & 0.9480 & 0.9320 & 0.9400 & 0.9500 \\
\hline O Rate & 0.0280 & 0.0360 & 0.0460 & 0.0420 & 0.0340 & 0.0260 & 0.0360 & 0.0500 & 0.0310 & 0.0500 & 0.0340 & 0.0220 \\
\hline MW & 0.9636 & 1.0470 & 0.8700 & 0.9105 & 0.9701 & 1.0153 & 0.9432 & 0.8829 & 0.9892 & 0.7969 & 0.9392 & 0.5223 \\
\hline SDW & 0.0367 & 0.0517 & 0.0634 & 0.0663 & 0.0000 & 0.0000 & 0.0211 & 0.0388 & 0.0358 & 0.0491 & 0.0358 & 0.2334 \\
\hline \multicolumn{13}{|c|}{$n=30$} \\
\hline B Rate & 0.0040 & 0.0120 & 0.0300 & 0.0220 & 0.0270 & 0.0180 & 0.0120 & 0.0160 & 0.0303 & 0.0240 & 0.0220 & 0.0160 \\
\hline C Rate & 0.9900 & 0.9460 & 0.9200 & 0.9280 & 0.9490 & 0.9580 & 0.9460 & 0.9420 & 0.9495 & 0.9440 & 0.9440 & 0.9540 \\
\hline O Rate & 0.0060 & 0.0420 & 0.0500 & 0.0500 & 0.0240 & 0.0240 & 0.0420 & 0.0420 & 0.0202 & 0.0320 & 0.0340 & 0.0300 \\
\hline MW & 0.7600 & 0.8978 & 0.7162 & 0.7324 & 0.7698 & 0.7872 & 0.7429 & 0.7159 & 0.7936 & 0.6995 & 0.7534 & 0.4305 \\
\hline SDW & 0.0200 & 0.0314 & 0.0362 & 0.0371 & 0.0000 & 0.0000 & 0.0199 & 0.0310 & 0.0208 & 0.0315 & 0.0208 & 0.1821 \\
\hline \multicolumn{13}{|c|}{$n=50$} \\
\hline B.Rate & 0.0000 & 0.0210 & 0.0160 & 0.0160 & 0.0220 & 0.0100 & 0.0280 & 0.0280 & 0.0240 & 0.0140 & 0.0240 & 0.0210 \\
\hline C Rate & 0.9980 & 0.9470 & 0.9300 & 0.9300 & 0.9500 & 0.9620 & 0.9480 & 0.9480 & 0.9500 & 0.9460 & 0.9500 & 0.9550 \\
\hline O Rate & 0.0020 & 0.0320 & 0.0540 & 0.0540 & 0.0280 & 0.0280 & 0.0240 & 0.0240 & 0.0260 & 0.0400 & 0.0260 & 0.0240 \\
\hline MW & 0.5740 & 0.6454 & 0.5589 & 0.5616 & 0.5835 & 0.5863 & 0.5300 & 0.5552 & 0.5931 & 0.5496 & 0.5431 & 0.3128 \\
\hline SDW & 0.0092 & 0.0158 & 0.0174 & 0.0175 & 0.0000 & 0.0000 & 0.0892 & 0.0259 & 0.0087 & 0.0056 & 0.0087 & 0.1598 \\
\hline
\end{tabular}


(Table 5). Continued.

\begin{tabular}{|c|c|c|c|c|c|c|c|c|c|c|c|c|}
\hline Critria & $\mathrm{CCl}$ & $\mathrm{FCl}$ & Clr & $\operatorname{Clr}(t)$ & $\mathrm{Clz}$ & $\mathrm{Clz}(\mathrm{t})$ & SKCl & NBoot & PBoot & FBoot & SKBoot & BCA \\
\hline \multicolumn{13}{|c|}{$n=100$} \\
\hline B Rate & 0.0000 & 0.0110 & 0.0320 & 0.0360 & 0.0140 & 0.0100 & 0.0140 & 0.0140 & 0.0200 & 0.0200 & 0.0210 & 0.0140 \\
\hline C Rate & 1.0000 & 0.9490 & 0.9440 & 0.9380 & 0.9520 & 0.9720 & 0.9490 & 0.9560 & 0.9560 & 0.9540 & 0.9560 & 0.9560 \\
\hline O Rate & 0.0000 & 0.0300 & 0.0240 & 0.0260 & 0.0340 & 0.0180 & 0.0370 & 0.0300 & 0.0240 & 0.0260 & 0.0230 & 0.0300 \\
\hline MW & 0.3989 & 0.4690 & 0.3979 & 0.3948 & 0.4061 & 0.4029 & 0.3812 & 0.3914 & 0.4251 & 0.3716 & 0.3851 & 0.2045 \\
\hline SDW & 0.0028 & 0.0053 & 0.0056 & 0.0055 & 0.0000 & 0.0000 & 0.0198 & 0.0178 & 0.0027 & 0.0050 & 0.0027 & 0.1999 \\
\hline
\end{tabular}

Notes: CCl - Classical confidence interval given in (2), FCl - Fisher confidence interval given in (3), Clr - Gorsuch and Lehman confidence interval 1 given in (4), $\mathrm{Clr}(\mathrm{t})$ - Gorsuch and Lehman confidence interval 2 given in (5), Clz - Gorsuch and Lehman confidence interval 3 given in (6), Clz(t) - Gorsuch and Lehman confidence interval 4 given in (7), SKCl -Shipra and Kibria proposed confidence interval given in (8), NBoot- Non-parametric confidence interval given in (9), PBoot- Parametric confidence interval given in (10), FBoot-Fisher bootstrap confidence interval given in (11), SKBoot - Shipra and Kibria bootstrap confidence interval given in (12) and BCA - Bootstrap bias corrected acceleration confidence interval given in (13).

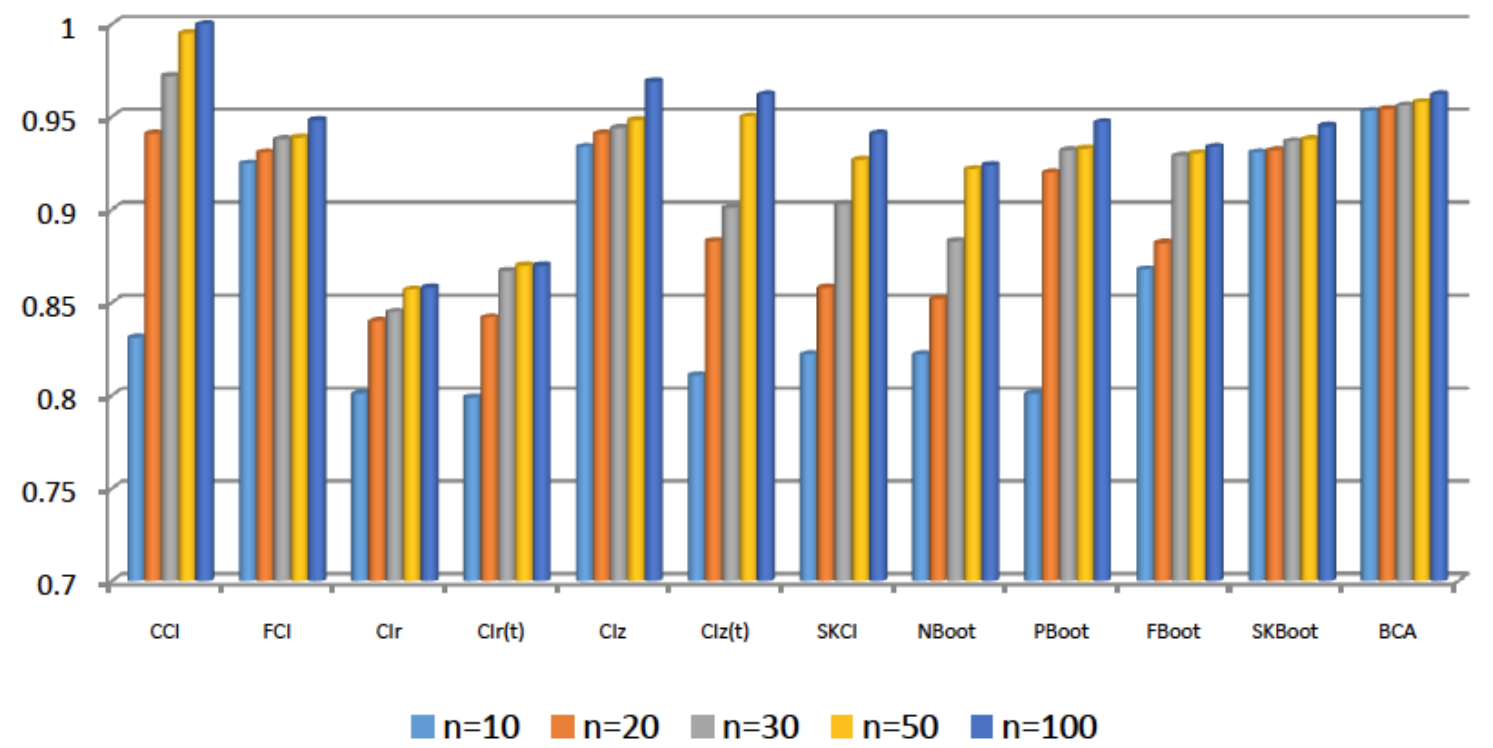

Figure 1: Coverage probability for various values of $n$ in case of bivariate normal distribution.

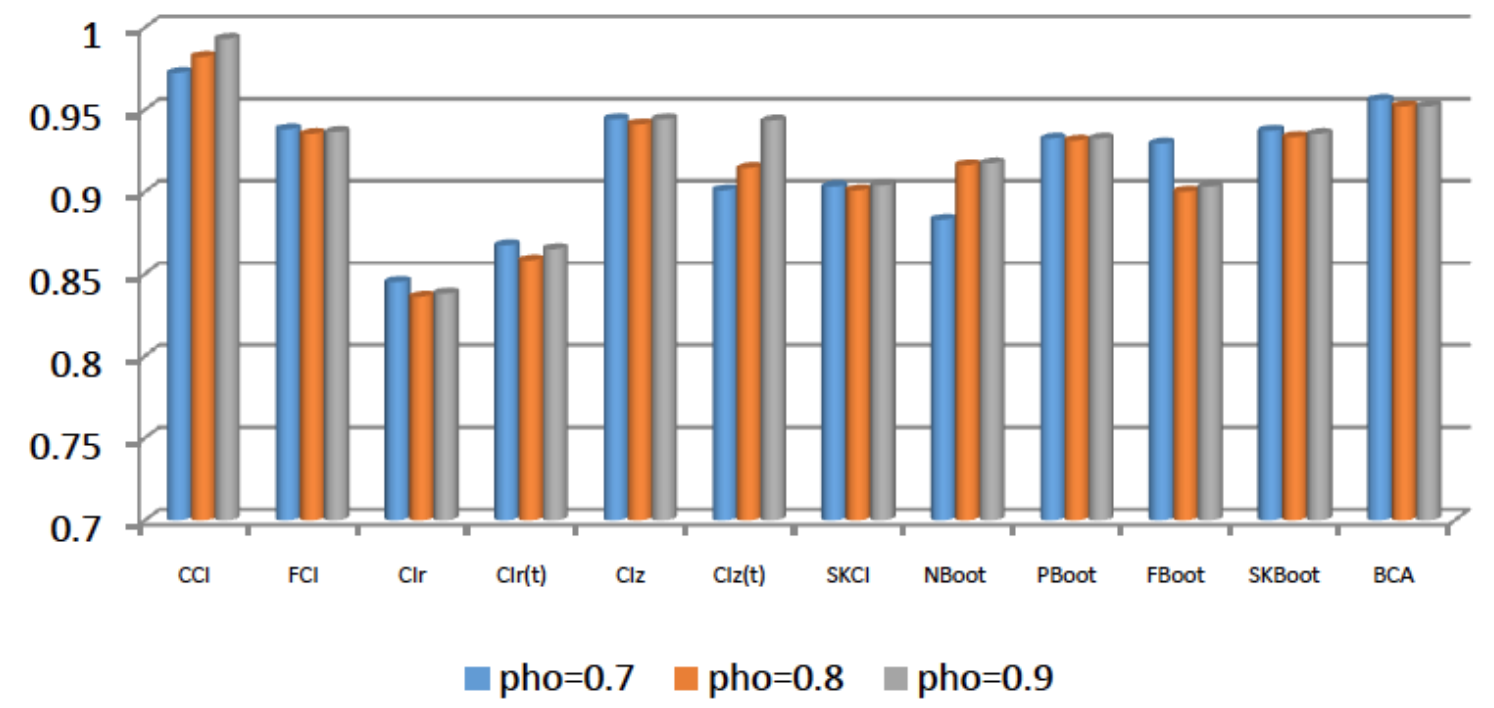

Figure 2: Coverage probability for various values of $\rho_{1}$ and $n=30$ in case of bivariate normal distribution. 


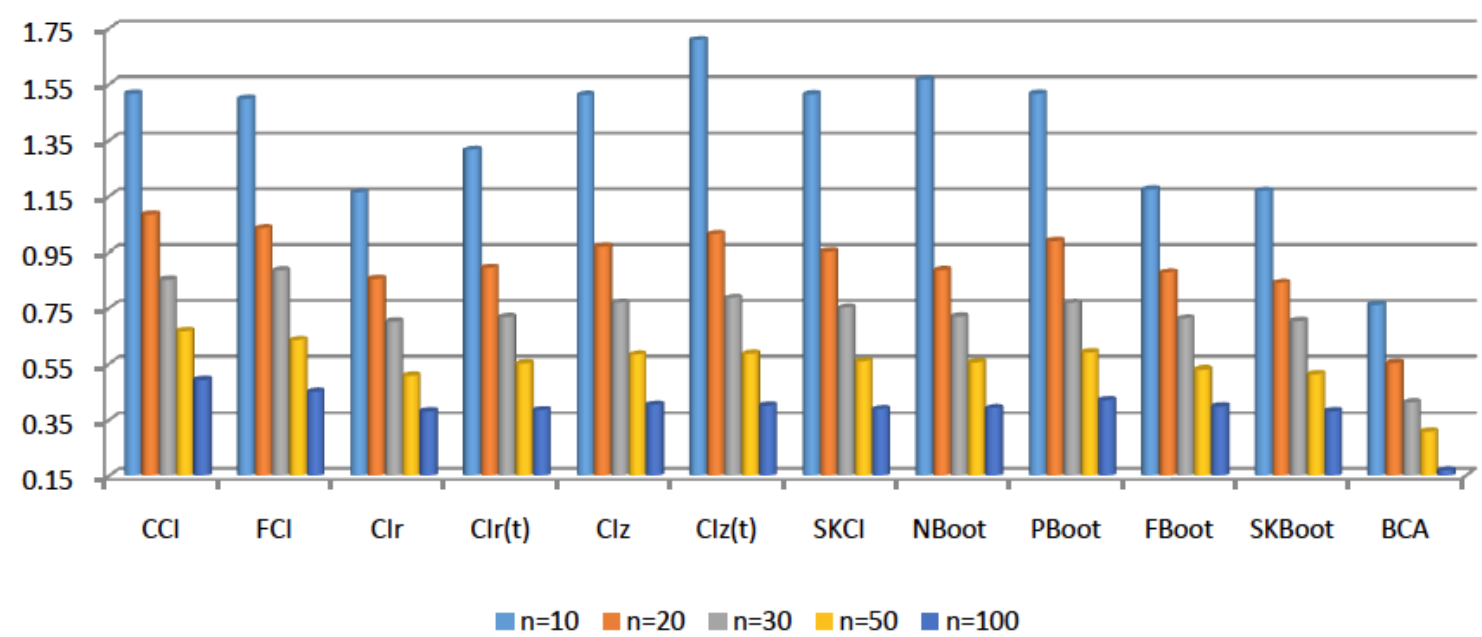

Figure 3: Mean widths for various values of $n$ and $\rho=0.9$ in case of bivariate normal distribution.

sizes in Figure 3 for $\rho_{1}=0.90$. We see that as the sample size $\mathrm{n}$ increases, mean widths are decreases. We found that BCA, Clr, SKBoot and FBoot have shorter mean widths compared to others.

Next we evaluated our considered Cls when data are generated from the bivariate lognormal distribution for various values of $n$ and $\rho_{1}$. To save space of the paper, only results for $\rho_{1}=0.7$, are reported in Table 4 (others simulation results are available upon request). Figure 4 shows that as the sample size increases, coverage probabilities are approaching to nominal level 0.95 except $\mathrm{CCl}, \mathrm{Clr}, \mathrm{Clr}(\mathrm{t})$ and $\mathrm{SKCl}$ intervals. In general $\mathrm{FCl}, \mathrm{Clz}$, SKBoot and BCA have higher cover rates as compare to others.

We have only tabulated simulation results when data are generated from the bivariate chi-square distribution in Table 5 to save space of the paper (others results are available upon request). In Figure 5, we have presented coverage probabilities for various values of $n$ and $\rho_{1}=0.7$. We observed that all methods but $\mathrm{CCl}, \mathrm{Clr}$ and $\mathrm{Clz}(\mathrm{t})$ have coverage probability very close to the nominal level 0.95 .

In general, from our simulation studies, we found that the coverage probabilities of intervals, BCA, SKBoot, $\mathrm{Clz}$ and $\mathrm{FCl}$ are close to nominal level 0.95, while BCA, CIr, FBoot and SKBoot have shorter mean widths as compare to others.

\section{APPLICATIONS}

This section considers two real life applications to illustrate the findings of the paper.

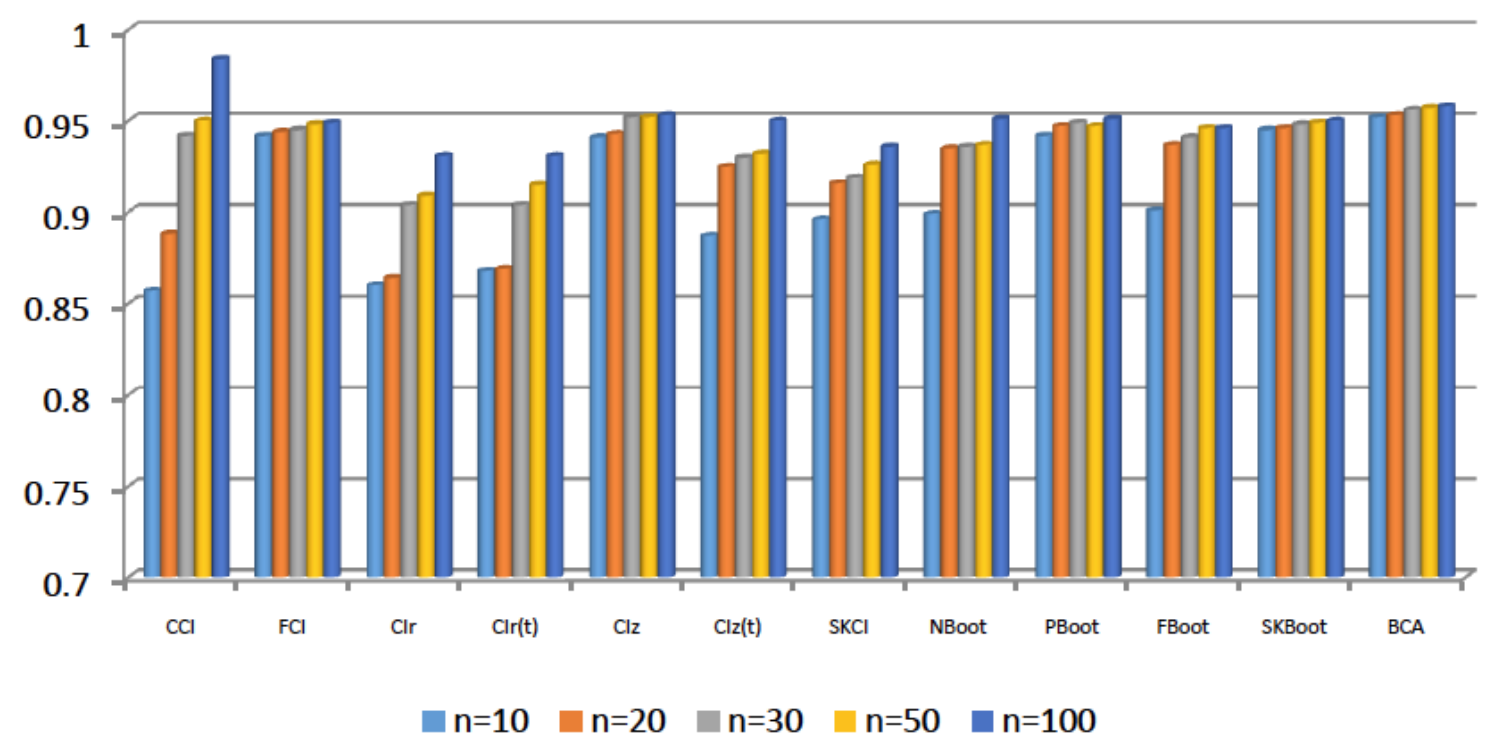

Figure 4: Coverage probability for various values of $n$ in case of bivariate lognormal distribution. 


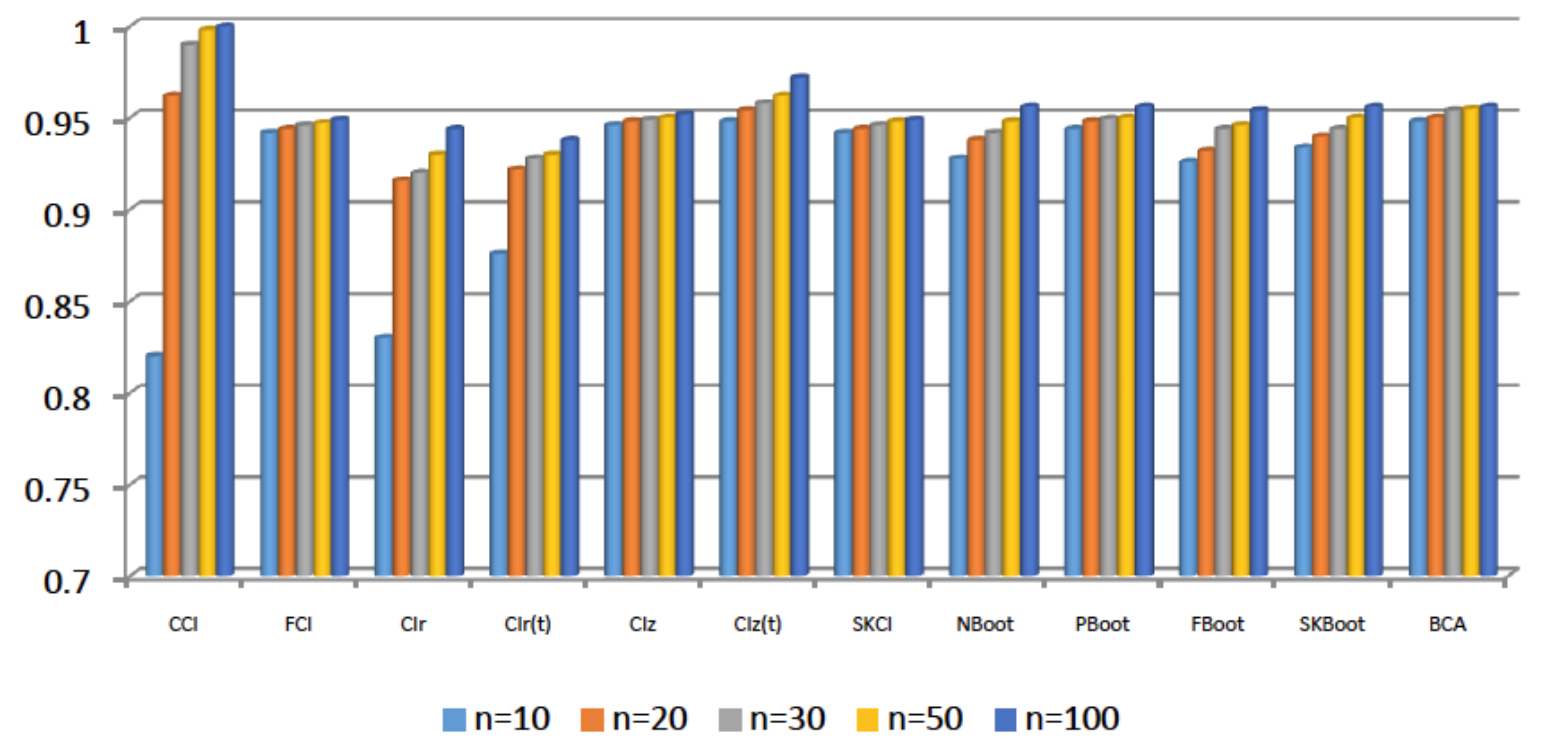

Figure 5: Coverage probability for various values of $n$ in case of bivariate chi-square distribution.

\subsection{Example 1}

The subjects of a study by Malec et al. (1991) [11] were 16 graduates of a comprehensive, post acute brain injury rehabilitation program. The researcher examined the relationship among a number of variables, including work outcome (scaled from 1 for unemployed to 5 , which represents competitive nonsheltered employment), score at time of period evaluation on the Portland Adaptability Inventory (PAI) and length of stay (LOS) in days. The following measurements on these three variables were collected: and FBoot when $y$ is correlated with $x_{1}$, while BCA has the shortest width followed by Clr, FBoot and SKBoot when y correlated with $x_{2}$.

\subsection{Example 2}

The objective of a study by Steinhorn and Green (1991) [12] was to determine whether the metabolic response to illness in children as measured by direct means is correlated with the estimated severity of illness. Subjects were 12 patients between the ages of 2 and 120 months with a variety illness including sepsis, bacterial meningitis and respiratory failure.

\begin{tabular}{|c|c|c|c|c|c|c|c|c|c|c|c|c|c|c|c|c|}
\hline $\begin{array}{c}y \text {-work } \\
\text { experience }\end{array}$ & 5 & 4 & 2 & 4 & 1 & 4 & 1 & 4 & 4 & 5 & 3 & 1 & 4 & 1 & 4 & 4 \\
\hline $\mathrm{x}_{1}$-LOS(days) & 67 & 157 & 242 & 255 & 227 & 140 & 179 & 258 & 85 & 52 & 296 & 256 & 198 & 224 & 126 & 156 \\
\hline $\mathrm{x}_{2}$-PAI PRE & 19 & 17 & 23 & 14 & 27 & 22 & 23 & 18 & 16 & 22 & 15 & 30 & 21 & 22 & 19 & 8 \\
\hline
\end{tabular}

We are interested to find confidence interval of the correlation coefficient between $y$ and $x_{1}$ and $y$ and $x_{2}$ using selected methods of this paper. To get an idea about the relation between $y$ and $x_{1}$ and $y$ and $x_{2}$, we draw scatter plots and presented them in Figures 6 and 7 respectively. We find that there is a negative relation between $y$ and $\left(x_{1}, x_{2}\right)$. The correlation between $y$ and $x_{1}$ is -0.5780 and $y$ and $x_{2}$ is -0.5969 , indicate negative relation between considered variables. The 95\% confidence intervals for $r$ between $y$ and $\left(x_{1}, x_{2}\right)$ are provided in Table 6.

We see from the Table 6 that the interval estimator BCA has the shortest width followed by CIr, SKBoot

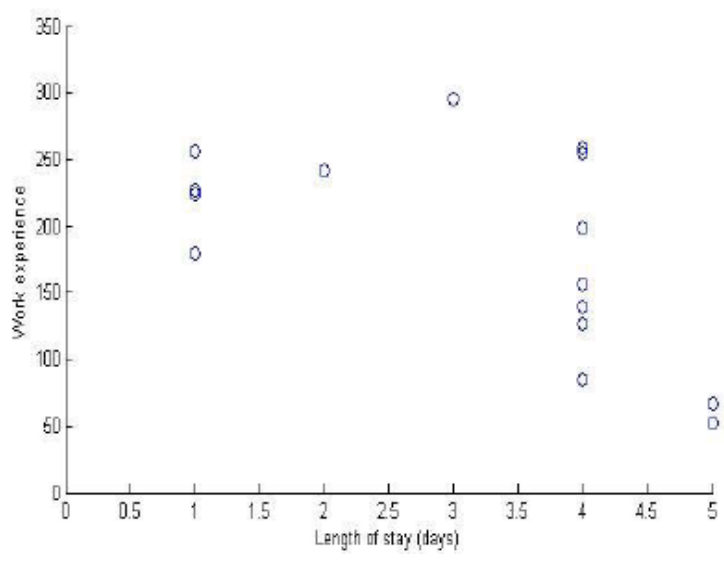

Figure 6: Scatter diagram y with $\mathrm{x}_{1}$. 


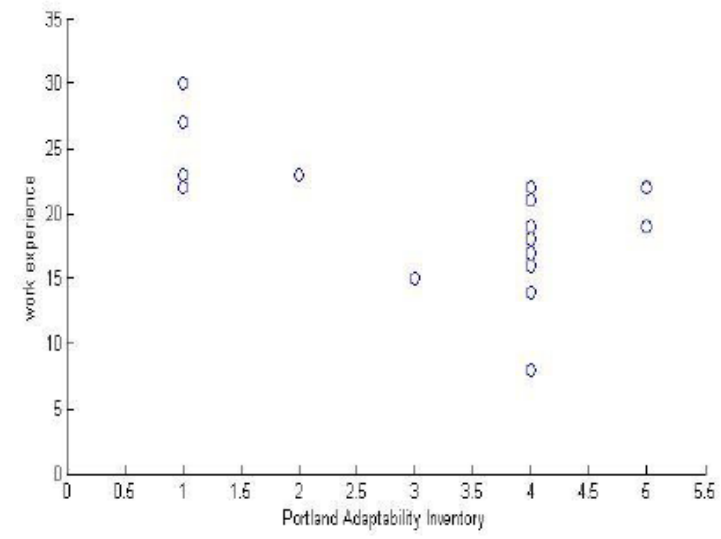

Figure 7: Scatter diagram y with $\mathrm{x}_{2}$.

Severity of illness was assessed by means of the Physiologic Stability Index (PSI) and the Rediatric Risk of Mortality scoring system (PRISM). The resulting measurements on these variables were as follows:

PRISM: 15, 27, 5, 23, 4, 6, 18, 15, 12, 1, 50, 9

PSI:14, 18, 4, 18, 12, 4, 17, 14, 11, 4, 63, 10

We want to find confidence intervals of correlation between PRISM and PSI. The scatter plot between PRISM and PSI in Figure 8 indicates a positive relation between PRISM and PSI. The 95\% confidence intervals of the correlation coefficient between PRISM and PSI of our selected methods are given in Table 7 . It appears from this table, overall BCA, SKBoot, Clr, FBoot, have the shorter widths compared to the rest.

From our studies, in general it can be concluded that the BCA is the best method, followed by SKBoot, FBoot, FCl, PBoot, Clz, SKCl, Clr, CCl, Clr(t), NBoot and $\mathrm{Cl} z(\mathrm{t})$. The above order is made based on the good coverage probability, small average width and size of samples. We believe that the simulation results along with applications will be helpful for practitioners to choose good methods to find confidence intervals for $\rho$.

\section{CONCLUSION}

This paper considers several methods for constructing a confidence interval estimator for the population correlation coefficient $\rho$. A simulation study has been conducted to compare the performance of the estimators. Data were randomly generated from several bivariate distributions, such as, bivariate normal, bivariate lognormal and bivariate chi-square with a range of sample sizes. Various evaluation criterions such as below rate, cover rate, over rate, mean widths and SD of the widths are considered to compare the performance of the estimators. Two real life applications are considered to illustrate the findings of the paper.

Overall, it appears that BCA performed the best followed by SKBoot, $\mathrm{Clz}$ and $\mathrm{FCl}$ when we consider the coverage probability close to the nominal level. BCA has the smallest average width followed by Clr, SKBoot and FBoot. It is evident from both simulation study and application that the BCA and SKBoot confidence intervals can be recommended for practitioners because these two intervals performed better than the rest of the estimators. It is also evident from our study that these two methods work well for small sample sizes although as compare to $\mathrm{CCl}$, these two methods are not so handy to compute. Our proposed method also performing reasonably well in the sense of good

Table 6: $95 \%$ confidence intervals for $\rho$

\begin{tabular}{|c|c|c|c|c|c|}
\hline \multicolumn{3}{|c|}{$r$ between $y$ and $x_{1}$} & \multicolumn{3}{|c|}{$r$ between $y$ and $x_{2}$} \\
\hline Methods & Cls & Width & Methods & Cls & Width \\
\hline $\mathrm{CCl}$ & $(-0.8457,-0.1102)$ & 0.7355 & $\mathrm{CCl}$ & $(-0.8568,-0.1369)$ & 0.7199 \\
\hline $\mathrm{FCl}$ & $(-0.8346,-0.1153)$ & 0.7193 & $\mathrm{FCl}$ & $(-0.8431,-0.1437)$ & 0.6994 \\
\hline Clr & $(-0.9219,-0.2341)$ & 0.6878 & Clr & $(-0.9293,-0.2644)$ & 0.6649 \\
\hline $\mathrm{CIr}(\mathrm{t})$ & $(-0.9445,-0.2115)$ & 0.7330 & $\mathrm{CIr}(\mathrm{t})$ & $(-0.9611,-0.2426)$ & 0.7185 \\
\hline $\mathrm{Clz}$ & $(-0.8379,-0.1043)$ & 0.7336 & $\mathrm{Clz}$ & $(-0.8563,-0.1328)$ & 0.7235 \\
\hline $\mathrm{Clz}(\mathrm{t})$ & $(-0.8484,-0.0681)$ & 0.7803 & $\mathrm{Clz}(\mathrm{t})$ & $(-0.8563,-0.0968)$ & 0.7595 \\
\hline SKCl & $(-0.8450,-0.1100)$ & 0.7350 & SKCl & $(-0.8515,-0.1380)$ & 0.7135 \\
\hline NBoot & $(-0.8975,-0.1140)$ & 0.7835 & NBoot & $(-0.8954,0.1211)$ & 0.7743 \\
\hline PBoot & $(-0.8527,-0.0965)$ & 0.7562 & PBoot & $(-0.8669,-0.1601)$ & 0.7068 \\
\hline FBoot & $(-0.8229,-0.1105)$ & 0.7124 & FBoot & $(-0.8475,-0.1677)$ & 0.6798 \\
\hline SKBoot & $(-0.8233,-0.1126)$ & 0.7107 & SKBoot & $(-0.8359,-0.1378)$ & 0.6981 \\
\hline $\mathrm{BCA}$ & $(-0.7945,-0.1167)$ & 0.6778 & $\mathrm{BCA}$ & $(-0.7410,-0.1390)$ & 0.6020 \\
\hline
\end{tabular}




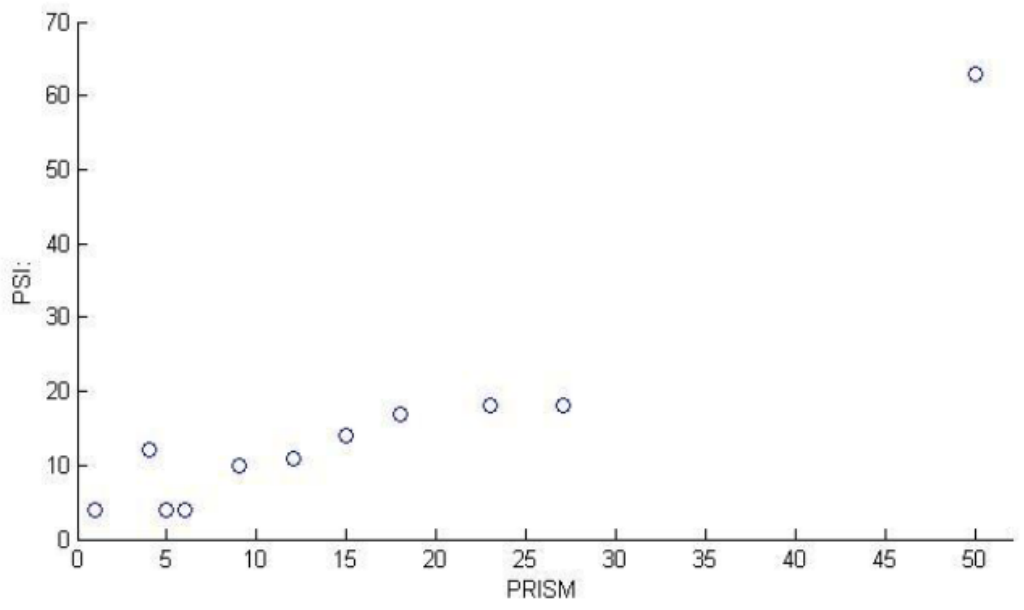

Figure 8: Scatter diagram PSI with PRISM.

coverage and shorter width. We expect that the results of this paper will be helpful for practitioners and researchers when they will choose to calculate confidence interval for the population correlation coefficient and formulate policies.

Table 7: $95 \%$ Confidence Intervals for $\rho$

\begin{tabular}{|c|c|c|}
\hline Methods & Cls & Width \\
\hline \hline $\mathrm{CCl}$ & $(0.6890,0.9737)$ & 0.2847 \\
\hline $\mathrm{FCl}$ & $(0.7734,0.9821)$ & 0.2097 \\
\hline $\mathrm{Clr}$ & $(0.8020,0.9910)$ & 0.1890 \\
\hline $\mathrm{Clr}(\mathrm{t})$ & $(0.7845,0.9881)$ & 0.2036 \\
\hline $\mathrm{Clz}$ & $(0.7782,0.9828)$ & 0.2046 \\
\hline $\mathrm{Clz}(\mathrm{t})$ & $(0.7504,0.9850)$ & 0.2346 \\
\hline $\mathrm{SKCl}$ & $(0.6980,0.9610)$ & 0.2630 \\
\hline $\mathrm{NBoot}$ & $(0.5140,0.8556)$ & 0.3416 \\
\hline $\mathrm{PBoot}$ & $(0.7039,0.9129)$ & 0.2090 \\
\hline FBoot & $(0.7838,0.9866)$ & 0.2028 \\
\hline $\mathrm{SKBoot}$ & $(0.8036,0.9891)$ & 0.1855 \\
\hline $\mathrm{BCA}$ & $(0.8945,0.9783)$ & 0.0838 \\
\hline
\end{tabular}

\section{ACKNOWLEDGEMENTS}

Author, B. M. Golam Kibria dedicates this paper to his Father-in-Law, Late Professor Mahbubul Haq for his love and inspiration, which, certainly inspired him to reach at his present position. Authors are thankful to the referee and Editor for their valuable comments and suggestions which certainly improved the quality and presentation of the paper.

\section{REFERENCES}

[1] Fisher RA. On the Probable Error of a Coefficient of Correlation Deduced from a Small Sample. Metron 1921; 1: 3-32.

[2] Von Storch H, Zwiers FW. Statistical Analysis in Climate Research. Cambridge University Press, Cambridge, UK 1999.

[3] Wilks DS. Statistical Methods in the Atmospheric Sciences. Academic Press, San Diego, CA 1995.

[4] Neff U, Burns SJ, Mangini A, Mudelsee M, Fleitmann D, Matter A. Strong Coherence between Solar Variability and the Monsoon in Oman between 9 and 6 kyrAgo. Nature 2001; 411: 290-293. http://dx.doi.org/10.1038/35077048

[5] Hall P, Martin MA, Schucany WR. Better Nonparametric Bootstrap Confidence Intervals for the Correlation Coefficient. Journal of Statistical Computation and Simulation 1989; 33: 161-172. http://dx.doi.org/10.1080/00949658908811194

[6] Student. The Probable Error of a Correlation Coefficient. Biometrika 1908; 6: 302-10. http://dx.doi.org/10.1093/biomet/6.2-3.302

[7] Fisher RA. Frequency Distribution of the Values of the Correlation Coefficient in Samples from an Indefinitely Large Population. Biometrica 1915; 10: 507-521. http://dx.doi.org/10.2307/2331838

[8] Gorsuch RL, Lehmann CS. Correlation Coefficient: Mean Bias and Confidence Interval Distortions. Journal of Methods and Measurement in the Social Science 2010; 1: 52-65.

[9] Efron B. Bootstrap Methods: Another Look at the Jack knife. Annals of Statistics 1979; 7: 1-26. http://dx.doi.org/10.1214/aos/1176344552

[10] Efron B, Tibshirani RJ. An Introduction to the Bootstrap, Chapman \& Hall, New York 1993. http://dx.doi.org/10.1007/978-1-4899-4541-9

[11] Malec JF, Ivnik RJ, Hinkeldey NS. Visual S;atial Learining test. Psychological Assessment 1991; 3: 82-88.

[12] Steinhorn DM, Green TP. Severity of Illness Correlates with Alternations in Energy Metabolism in the Pediatric Intensive Care Unit. Critical Care Medicine 1991; 19: 1503-1509. http://dx.doi.org/10.1097/00003246-199112000-00011 